\title{
Fluid Flow and Solid/Fluid Suspensions Flow in 3-D Packed Beds of Spheres: the Effect of Periodicity of Fixed Beds
}

\author{
A. Serrenho ${ }^{1,2 a}$ and A.F. Miguel ${ }^{\star 1,3, b}$ \\ ${ }^{1}$ Geophysics Centre of Évora, Rua Romao Ramalho 59, 7000-671 Évora, Portugal \\ ${ }^{2}$ Department of Civil Engineering, National University of Ireland, Galway, Ireland \\ ${ }^{3}$ Department of Physics, University of Évora, PO Box 94, 7002-554 Évora, Portugal \\ aana.serrenho@gmail.com , ${ }^{\mathrm{b}}$ afm@uevora.pt
}

Keywords: Packed beds, fluid flow, solid/fluid suspensions flow, permeability, inertial factor, penetration efficiency, deposition

\begin{abstract}
A 3-D numerical study is performed to investigate the effects of periodicity (geometry) on flow of fluid and on flow of solid/fluid suspensions in packing arrangements of fixed beds of spheres. The porosity is fixed at 0.58 and the following packing arrangements are studied: simple cubic, face-centered cubic, hexagonal, rhomboedric hexagonal and tetragonal. Simulations are carried out at Reynolds numbers ranging from 0.1 and 50, and using solid suspensions with different sizes $\left(0.2,2\right.$ and 10 micron) and densities $\left(200\right.$ and $\left.2000 \mathrm{~kg} / \mathrm{m}^{3}\right)$. The effect of the periodicity on fluid flow characteristics (permeability and inertia parameter) and on the penetration efficiency of solid suspensions within the packed beds is analyzed and quantified.
\end{abstract}

\section{Introduction}

A set of non-overlapping solid objects is called a packing. The interest in packing structures dates back to early times when the grain packings called "heaps" were the first things that were ever measured in ancient civilizations both for trading or taxes collection [1]. Packing structures has also fascinated scientists for centuries. They have served as models to understand the structure of liquid and crystal states of matter, heterogeneous materials and granular media [2-5].

Sphere packing is a very common type of particle packing and has been studied by biologists, materials scientists, engineers, chemists, and physicists [5,6].. Packings of identical spheres are commonly used for several operations such as in granular media for fluid flow, and for processes involving absorption, adsorption of a solute, distillation, filtration and separation [7,8]. Despite its importance and long history [7], there are some fundamental issues concerning packings of spheres that remain elusive, including the precise effect of packing arrangements on flow of solid/fluid suspensions (e.g., aerosol transport and deposition). Therefore, the effect of periodicity (geometry) is of fundamental importance in a wide variety of environmental, biological and technological processes.

Nearly half millennium ago, Johannes Kepler [9] stated that the face-centered cubic packing is the way pack identical spheres together in the densest possible way (i.e., porosity of $1-\pi / 18^{1 / 2}(\sim$ $0.260)$ and packing density $\left.\pi / 18^{1 / 2}\right)$. This arrangement found in cannonballs piled at war memorials or fruit arrangements was called the Kepler conjecture [10]. The packing structure can also be presented within different arrangements (e.g., cubic, hexagonal, orthorhombic, etc.). In case of identical spheres, together in the densest possible way, the packing density of these structures varies between $\pi / 18^{1 / 2}$ (rhomboedric hexagonal or face-centered cubic) and $\pi / 6$ (simple cubic).

The characteristics of packing affects local fluid, mass and heat transport. Therefore, the packing arrangement is directly relevant to macroscopic key parameters such as the fluid permeability and the particle penetration coefficient [7,11]. In this paper, we study the effect of periodicity (geometry) in fixed beds of spheres (i.e., non-overlapping solid objects) both on flow of fluid and flow of solid/fluid suspension via direct numerical simulation. The following packing arrangements are studied: simple cubic, face-centered cubic, hexagonal, rhomboedric hexagonal and tetragonal. To obtain only the effect of periodicity, the spheres diameter and the porosity are the same for all 
theses packing arrangements. The fluid dynamics of the suspending fluid is solved using NavierStokes equations and solid suspensions by means the force balance on the particle written in a Lagrangian reference frame.

\section{Numerical simulation: model of packed beds of spheres and gas-solid suspension flows}

Model of Packed Beds of Spheres. We simulate fluid and solid/fluid suspension flows through 5 packing structures in fixed spheres: models SCU (simple cubic), FCC (face-centered cubic), ORT (orthogonal), HEX (hexagonal), RHE (rhomboedric hexagonal) and TET (tetragonal). These structures are constructed with spheres of same diameter and have the same porosity (Table 1).

What is the impact of periodicity (geometry) on the fluid flow? and on the solid/fluid suspensions flow? The answers are provided via numerical simulation described next.

3D Numerical Determination of Fluid Flow. In the limit of incompressible flow, steady state fluid flow is described by the equations

$$
\begin{aligned}
& \rho(\mathrm{v} . \nabla \mathrm{v})=-\nabla \mathrm{p}+\mu \nabla^{2} \mathrm{v} \\
& \nabla . \mathrm{v}=0
\end{aligned}
$$

where $\mathrm{v}$ represents the local velocity, $\mathrm{p}$ is the pressure, and $\rho$ and $\mu$ are, respectively, the fluid density and the dynamic viscosity. To numerically solve these equations in $3 \mathrm{D}$, we used the code Fluent [12] which is based on a finite volume scheme. The fluid velocity must vanish at fluid-solid spheres interfaces and velocity is prescribed at the inlet of each packing arrangement while outflow boundary conditions are set at the exit.

Grids were generated in Gambit [12] for all packing arrangements. The cell size of the grid where high gradients of velocities are expected is varied to ensure a grid independent solution. Grids with 49365-60718 cells and 12615-15150 nodes are found to be appropriate for present study.

\begin{tabular}{|c|c|c|c|c|}
\hline Packing Mode & & Arrangement & Packing Density & Porosity \\
\hline simple cubic & $S C U$ & & \multirow{6}{*}{0.42} & \multirow{6}{*}{0.58} \\
\hline face-centered cubic & $F C C$ & & & \\
\hline Orthogonal & ORT & & & \\
\hline Hexagonal & $H E X$ & & & \\
\hline rhomboedric hexagonal & RHE & & & \\
\hline Tetragonal & TET & & & \\
\hline
\end{tabular}

Table 1 - Ordered packings of spheres with same sphere diameter and porosity.

The permeability and the inertia coefficient of each packing structure are calculated by applying the so-called Forchheimer equation [7,13]

$$
\frac{\Delta \mathrm{p}}{\mathrm{L}}=\frac{\mu}{\mathrm{K}} \mathrm{u}+\rho \alpha \mathrm{u}^{2}
$$

Here $\mathrm{u}$ is the superficial fluid velocity, $\mathrm{K}$ is the permeability, $\Delta \mathrm{p} / \mathrm{L}$ is the pressure drop and $\alpha$ is an inertial coefficient. Eq. (3) is not a purely empirical expression since it can be derived by an adequate average of the Navier-Stokes equation for steady and incompressible laminar flow of a Newtonian fluid in a rigid porous medium [14]. For convenience, Eq. (3) is often rearranged in a 
friction factor-Reynolds number dimensionless correlation. Here, following a suggestion of Miguel [15] is rearranged in a friction factor- Forchheimer number dimensionless correlation as follows

$$
\mathrm{f}_{\alpha}=\frac{1}{\mathrm{Fo}_{\mathrm{K}}}+1
$$

where $f_{\alpha}$ is the friction factor $\left(=\Delta p /\left(\rho \alpha \mathrm{Lu}^{2}\right)\right), \mathrm{Fo}_{\mathrm{K}}$ is the Forchheimer number based on permeability $\left(=\lambda \mathrm{Re}_{\mathrm{K}}\right), \mathrm{Re}_{\mathrm{K}}$ is the Reynolds number based on permeability $\left(=\rho \mathrm{K}^{1 / 2} / \mu\right)$ and $\lambda$ is the dimensionless inertia parameter $\left(=\alpha \mathrm{K}^{1 / 2}\right)$. Eq. (4) may be used to correlate data from a large variety of packed structures and a broad range of flow conditions.

Numerical Solid-Suspension Dynamics. There are two important aspects of solid/fluid suspension dynamics: the motion of the particles in the fluid and the interaction of the particles with the solid spheres of the packing arrangement. Once the steady flow field is obtained, the motion of the particles in the fluid may be pursued with the Lagrangian type equation of motion [11]

$$
\frac{d u_{p}}{d t}=\frac{1}{\rho_{p}}\left[\frac{18 \mu}{d_{p}^{2}} c_{g}\left(u-u_{p}\right)+\frac{1}{2} \rho \frac{d}{d t}\left(u-u_{p}\right)+\xi \rho\left(\frac{216 \pi \mu \sigma_{B} T}{\pi d_{p}^{5} c_{c} \Delta t}\right)^{1 / 2}\right]
$$

with $c_{g}=\frac{c_{d} \rho d_{p}}{24 \mu}\left|u_{p}-u\right|$ for micrometer and $c_{g}=\frac{1}{c_{c}}$ for submicrometer particles [7], $u_{p}$ is particle velocity, $\mathrm{c}_{\mathrm{d}}$ is drag coefficient, $\mathrm{c}_{\mathrm{c}}$ is the Cunningham slip correction factor [7], $\xi$ is the zero-mean unit-variance-independent Gaussian random number, $\sigma_{B}$ is the Boltzmann constant, $\rho_{p}$ is the particle density, and $\mathrm{T}$ is the absolute fluid temperature. Here the first right-hand term represents the drag force, the second right-hand term is the lift force due to shear, the third right-hand term denotes a force arising from Brownian collisions and the fourth right-hand term represents the effect of force of gravity on the particle. This motion of the particles in the fluid is also solved by the code Fluent [12]. The components of random force (third right-hand term) are evaluated at each time step. In this study, the particle is trapped at the solid spheres of the packing arrangement at first collision.

Particles (density 200 and $2000 \mathrm{~kg} / \mathrm{m}^{3}$, diameters between 0.2 and $10 \mu \mathrm{m}$ ) are released at the inlet of the packing structure cavity and tracked through the structure until they are trapped on the solid spheres or escape through the outlet of the structure. The particles penetration efficiency, $\Pi$, is evaluated using a particle counting technique, and defined as the ratio of outlet, $\mathrm{n}_{\text {out }}$, and inlet particle number, $\mathrm{n}_{\mathrm{inl}}$,

$$
\Pi=\frac{\mathrm{n}_{\text {out }}}{\mathrm{n}_{\mathrm{inl}}}
$$

For the sake of convenience, the results are presented in terms of penetration efficiency per number of solid spheres, $\Pi_{N}$, given by

$$
\Pi_{\mathrm{N}}=\frac{\Pi}{\mathrm{N}_{\text {spheres }}}
$$

where $\mathrm{N}_{\text {spheres }}$ is the total number of solid spheres that compose the packing structure.

\section{Results and Discussion}

First, we have performed complete 3D numerical simulations of air flow through packing structures, composed by spheres of diameter $\mathrm{d}_{\mathrm{esf}}\left(\mathrm{d}_{\mathrm{esf}}=\right.$ constant $)$, that are depicted in Table 1. Fig. 1 shows the flow pathlines for the packing structures subjected to low Reynolds conditions $(\operatorname{Re}=0.2)$ and high Reynolds conditions $(\mathrm{Re}=40)$. As shown in this figure, the situation is different at low and high Re: the predominant viscous forces in the momentum transport through the void geometry generates a more uniform "pathlines" velocities at $\mathrm{Re}=0.2$, whereas at $\mathrm{Re}=40$ "channels" of air flow are generated due to the relevant contribution of inertial forces. 


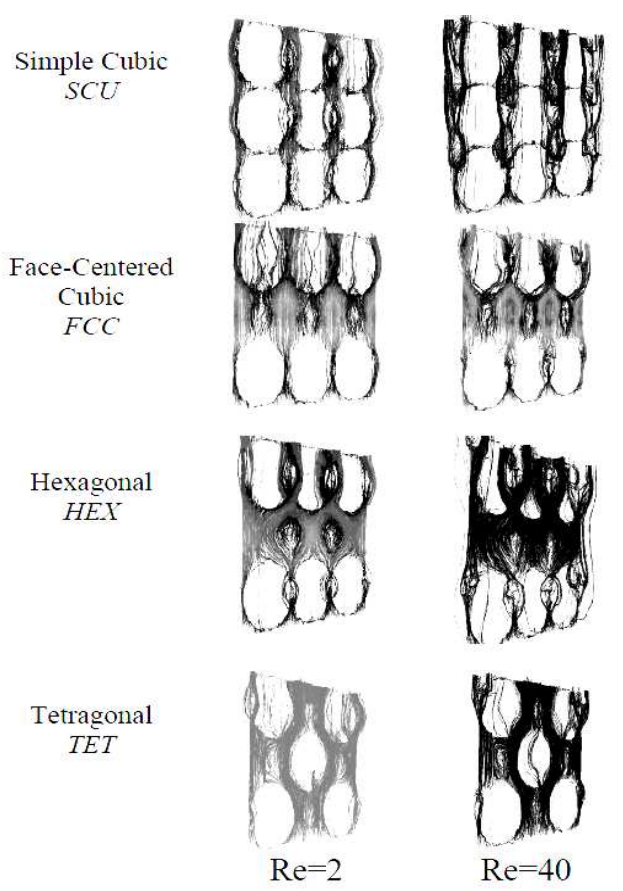

Figure 1 - Flow pathlines within the packing arrangements (longitudinal section).

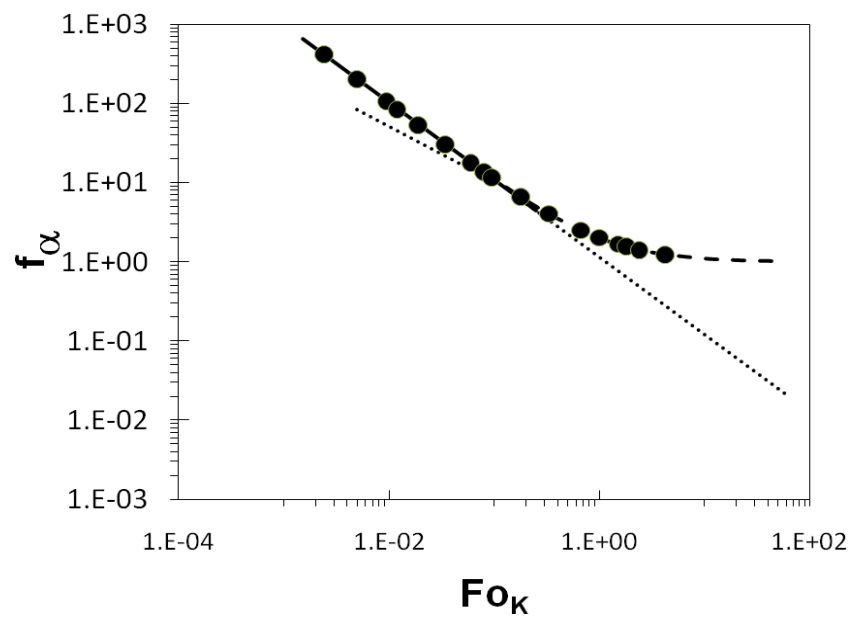

Figure 2 - The friction factor $\left(f_{\alpha}\right)$ versus the Forchheimer number $\left(\mathrm{Fo}_{\mathrm{K}}\right)$ : solid and dashed lines represent Eq. 4, solid line best fits Darcy regime, dashed line best fits nonlinear regime, and • represents results of the numerical simulations

After simulating and averaging the overall pressure drops for different inlet velocities, we estimated the coefficients $\mathrm{K}$ and $\alpha$. Eq. (3) was successfully correlated with our numerical data and the permeability and the inertial coefficient were obtained from the best fit (Table 2). Although both the porosity and diameter of spheres are the same, Table 2 shows that the permeability and the inertial coefficient are not the same for the packing structures. They fall into the range of $1.82 \times 10^{-8}$ $3.4 \times 10^{-8} \mathrm{~m}^{2}$ and $1650-2525 \mathrm{~m}^{-1}$, respectively. Only the following packings arrangements present very similar parameters: FCC and TET, RHE and ORT, and SCU and HEX. Notice that SCU and HEX detach from the others arrangements because they present the highest permeability and the lowest inertial coefficient. Packing models FCC, TET, RHE and ORT show permeabilities within the same range of magnitude $\left(\sim 2 \times 10^{-8} \mathrm{~m}^{2}\right)$.

Table 2 - Ordered packings of spheres: permeability and inertial coefficient.

\begin{tabular}{|c|c|c|c|c|}
\hline \multicolumn{2}{|l|}{ Packing Model } & Porosity & Permeability & Inertial Coefficient \\
\hline simple cubic & $S C U$ & \multirow{6}{*}{0.58} & 3.40 & 1650 \\
\hline face-centered cubic & $F C C$ & & 1.82 & 2525 \\
\hline Orthogonal & ORT & & 2.24 & 2241 \\
\hline Hexagonal & $H E X$ & & 3.20 & 1795 \\
\hline rhomboedric hexagonal & RHE & & 2.12 & 2277 \\
\hline Tetragonal & TET & & 1.93 & 2496 \\
\hline
\end{tabular}

In Fig. 2, we show both the results obtained with Eq. (4) and from the simulations performed with the packing arrangements. As shown in this figure, Eq. (4) is in agreement with our data and the transition from linear (Darcy's law) to nonlinear (inertial) behavior is in the range $0.08<\mathrm{Fo}_{\mathrm{K}}<$ 0.7 .

The penetration efficiency per number of solid spheres is documented in Table 3 . It is seen that, when the Reynolds number increases the probability to escape is higher but the size and the density of the particles don't have any particular effect on $\Pi_{\mathrm{N}}$. The situation changes drastically for big size and heavy particles under high Reynolds conditions. For particles with size $10 \mu \mathrm{m}$ and density 2000 
$\mathrm{kg} / \mathrm{m}^{3}\left(\operatorname{Re}_{\mathrm{D}}=40\right)$, the penetration efficiency per number of solid spheres becomes lower for packing structures FCC, RHE and TET, while it becomes higher for SCU and HEX. This observation can be explained based on Fig. 1. Big size and heavy particles (i.e., particles with large inertia) have a better chance to get escape from packing structures SCU and HEX due to the existence of well defined preferential channels of fluid flow that enable particles to cross these structures without contact with solid spheres.

Table 3 - Penetration efficiency per solid sphere $\Pi_{N}$

\begin{tabular}{|c|c|c|c|c|c|c|c|c|c|c|c|c|}
\hline \multirow{6}{*}{$\begin{array}{c}\text { Packing } \\
\text { Model }\end{array}$} & \multicolumn{6}{|c|}{ particles density $200 \mathrm{~kg} / \mathrm{m}^{3}$} & \multicolumn{6}{|c|}{ particles density $2000 \mathrm{~kg} / \mathrm{m}^{3}$} \\
\hline & \multicolumn{6}{|c|}{$\operatorname{Re}_{\mathrm{D}}\left(\operatorname{\rho ud}_{\mathrm{es}} / \mu\right)$} & \multicolumn{6}{|c|}{$\operatorname{Re}_{\mathrm{D}}\left(\operatorname{\rho ud}_{\mathrm{es}} / \mu\right)$} \\
\hline & \multicolumn{3}{|c|}{0.2} & \multicolumn{3}{|c|}{40} & \multicolumn{3}{|c|}{0.2} & \multicolumn{3}{|c|}{40} \\
\hline & \multicolumn{3}{|c|}{ Particles size $(\mu \mathrm{m})$} & \multicolumn{3}{|c|}{ Particles size $(\mu \mathrm{m})$} & \multicolumn{3}{|c|}{ Particles size $(\mu \mathrm{m})$} & \multicolumn{3}{|c|}{ Particles size $(\mu \mathrm{m})$} \\
\hline & 0.2 & 2 & 10 & 0.2 & 2 & 10 & 0.2 & 2 & 10 & 0.2 & 2 & 10 \\
\hline & \multicolumn{3}{|c|}{$\Pi_{N} 10^{-2}$} & \multicolumn{3}{|c|}{$\Pi_{N} 10^{-2}$} & \multicolumn{3}{|c|}{$\Pi_{N} 10^{-2}$} & \multicolumn{3}{|c|}{$\Pi_{N} 10^{-2}$} \\
\hline SCU & 2.5 & 2.5 & 2.5 & 2.8 & 2.9 & 3.1 & 2.5 & 2.5 & 2.6 & 2.9 & 3.0 & 3.2 \\
\hline FCC & 2.7 & 2.7 & 2.8 & 3.0 & 3.1 & 3.2 & 2.7 & 2.7 & 2.7 & 3.1 & 3.2 & 2.3 \\
\hline HEX & 2.7 & 2.7 & 2.8 & 3.0 & 3.1 & 3.2 & 2.7 & 2.8 & 2.8 & 3.1 & 3.1 & 3.5 \\
\hline RHE & 2.8 & 2.8 & 2.8 & 3.2 & 3.2 & 3.3 & 2.7 & 2.8 & 2.8 & 3.2 & 3.4 & 2.6 \\
\hline TET & 2.7 & 2.7 & 2.8 & 3.0 & 3.1 & 3.1 & 2.7 & 2.7 & 2.8 & 3.1 & 3.2 & 2.0 \\
\hline
\end{tabular}

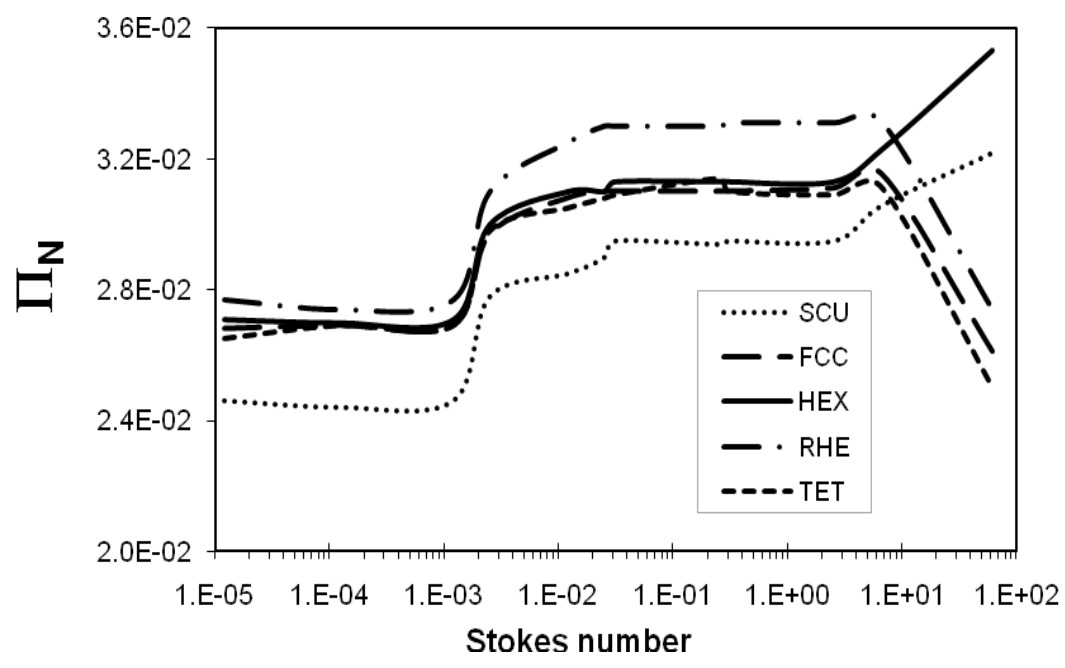

Figure 3 - The penetration efficiency per number of solid spheres $\left(\Pi_{\mathrm{N}}\right)$ versus the Stokes number (Eq. 8).

The penetration efficiency per number of solid spheres is also depicted in Fig. 3 in terms of Stokes number expressed by

$$
\mathrm{St}=\frac{\rho_{\mathrm{p}} \mathrm{d}_{\mathrm{p}}^{2} \mathrm{uc}_{\mathrm{c}}}{18 \mu \mathrm{d}_{\mathrm{esf}}}
$$


where St is the Stokes number and $\mathrm{c}_{\mathrm{c}}$ is the Cunningham slip correction factor [7]. According to Fig. 3 we come to the conclusion that the influence of the Stokes number in $\Pi_{N}$ is negligible for $10^{-5} \leq \mathrm{St}<10^{-3}$. For $10^{-3} \leq \mathrm{St}<50, \Pi_{\mathrm{N}}$ increases with $\mathrm{St}$ (this dependence is more noteworthy for $10^{-3} \leq \mathrm{St}<0.2$ ). The plot also reveals that for $\mathrm{St} \geq 50$, packing arrangements SCU and HEX experience an abrupt increase of $\Pi_{N}$ but FCC, RHE and TET undergo an abrupt decrease of $\Pi_{N}$. Therefore, the penetration efficiency variation profile of FCC, RHE and TET is different of the profile of SCU and HEX. This finding is in agreement with the flow results depicted in Table 2.

\section{Conclusion}

The analysis carried out in this paper has shown how periodicity (geometry) influences the fluid flow and the flow of solid suspensions in packing structure composed by fixed beds of spheres (porosity constant). Simulations carried out at Reynolds numbers ranging from 0.1 and 50 and solid suspensions of size 0.2, 2 and 10 micron show that: $(i)$ packing arrangements SCU and HEX have permeabilities $\sim 3 \times 10^{-8} \mathrm{~m}^{2}$ and FCC, TET, RHE and ORT of $\sim 2 \times 10^{-8} \mathrm{~m}^{2}$; (ii) packing arrangements SCU and HEX present inertial coefficients of 1650-1795 $\mathrm{m}^{-1}$ and the arrangements FCC, TET, RHE and ORT of 2241-2525 $\mathrm{m}^{-1}$, (iii) the transition from linear (Darcy's law) to nonlinear (inertial) fluid flow behavior is in the range of $0.08<\mathrm{Fo}_{\mathrm{K}}<0.7$, and (iv) big size and heavy particles have a better chance to get escape from packing arrangements SCU and HEX than from FCC, RHE and TET.

\section{References}

[1] J.D. Bernal: Liquids: Structure, Properties, Solid Interactions (edited by T. J. Hughel, 25-50, Elsevier, New York, 1965)

[2] A.G. Monroe and D.I. Gaffee: Nature Vol. 178 (1956), p. 197

[3] T.S. Majmudar, T.S. and R.P. Behringer: Nature Vol. 435 (2005), p. 1079

[4] D. Wen, and Y. Ding: Chem. Eng. Sci. Vol. 61 (2006), p. 3532

[5] S. Torquato, S. and Y. Jiao: Phys. Rev. E Vol. 80 (2009), p. 041104

[6] C.J. Geankoplis: Transport Process and Unit Operations (Prentice Hall, New Jersey, 2003).

[7] A. Bejan, I. Dincer, S. Lorente, A.F. Miguel and A.H. Reis: Porous and Complex flow Structures in Modern Technologies (Springer, New York, 2004)

[8] N. Kolev: Packed Bed Columns: For absorption, desorption, rectification and direct heat transfer (Elsevier, New York, 2006)

[9] J. Kepler: The Six-Cornered Snowflake (Oxford University Press, Oxford, 1966).

[10] D. Mackenzie: Am. Scientist Vol. 86 (1998), p. 1

[11] A. Serrenho and A.F. Miguel: J. Porous Med. Vol. 12 (2009), p. 1129

[12] Fluent: Fluent 6 User's Guide (Fluent Inc., 2003)

[13] A.F. Miguel: Energ. Build. Vol. 28 (1998), p. 63

[14] A.F. Miguel, van de Braak, N.J., Silva, A. M., and Bot, G. P. A: Journal of Wind Eng. and Ind. Aerodynamics Vol. 89 (2001) p. 45

[15] A.F. Miguel: Computational Analysis of the Role of Permeability and Inertia on Fluid Flow Through Porous Media (in Recent Progresses in Chemical Engineering, edited by J.M.P.Q. Delgado, chapter 1, 1-19, Studium Press LLC, Houston, 2010). 